\begin{tabular}{|c|l|}
\hline Title & $\begin{array}{l}\text { Electrical injection of spin-polarized electrons and electrical detection of dynamic nuclear polarization using a Heusler } \\
\text { alloy spin source }\end{array}$ \\
\hline Author(s) & A kiho, Takafumi; Shan, Jinhai; Liu, Hong-xi; Matsuda, Ken-ichi; Y amamoto, Masafumi; Uemura, Tetsuya \\
\hline Citation & $\begin{array}{l}\text { Physical Review B, 87(23), 235205 } \\
\text { https://doi.org/10.1103/PhysRevB.87.235205 }\end{array}$ \\
\hline Issue Date & 2013-06-17 \\
\hline Doc URL & http://hdl.handle.net/2115/53085 \\
\hline Rights & O2013A merican Physical Society \\
\hline Type & article \\
\hline File Information & PhysRevB.87.235205.pdf \\
\hline
\end{tabular}

Instructions for use 


\title{
Electrical injection of spin-polarized electrons and electrical detection of dynamic nuclear polarization using a Heusler alloy spin source
}

\author{
Takafumi Akiho, Jinhai Shan, Hong-xi Liu, Ken-ichi Matsuda, Masafumi Yamamoto, and Tetsuya Uemura* \\ Division of Electronics for Informatics, Hokkaido University, Sapporo 060-0814, Japan \\ (Received 25 January 2013; revised manuscript received 21 May 2013; published 17 June 2013)
}

\begin{abstract}
We demonstrated electrical spin injection from a half-metallic Heusler alloy $\mathrm{Co}_{2} \mathrm{MnSi}$ electrode into a GaAs channel through observation of a spin-valve signal and a Hanle signal in the four-terminal nonlocal geometry. Furthermore, we electrically detected a nuclear field acting on electron spins, which was produced by the dynamic nuclear polarization, through observation of transient oblique Hanle signals. Samples with a $\mathrm{Co}_{2} \mathrm{MnSi}_{\text {spin source }}$ exhibited higher spin-injection efficiency and a larger nuclear field compared to samples with a $\mathrm{Co}_{50} \mathrm{Fe}_{50}$ spin source, suggesting that the spin polarization of $\mathrm{Co}_{2} \mathrm{MnSi}$ is higher. This higher polarization is promising for realizing future spintronic devices and for understanding spin interactions as well as spin-dependent transport properties in a semiconductor channel.
\end{abstract}

DOI: 10.1103/PhysRevB.87.235205

PACS number(s): 72.25.Hg, 72.25.Dc, 72.25.Mk

\section{INTRODUCTION}

The injection and detection of spin-polarized electrons using ferromagnet/semiconductor heterojunctions have attracted much interest for creating viable spintronic devices featuring nonvolatile, reconfigurable logic functions and ultralow power consumption. The spin injection from a ferromagnetic electrode of $\mathrm{Fe}$ or $\mathrm{CoFe}$ into $\mathrm{GaAs}, \mathrm{Si}$, or $\mathrm{Ge}$ has been observed optically in spin light-emitting diodes (spin-LED ${ }^{1-5}$ ) and also electrically in lateral spin-transport devices through nonlocal geometry. ${ }^{6-12}$ Lou et al. demonstrated electrical spin injection in $\mathrm{Fe} / \mathrm{GaAs}$ Schottky tunnel junctions through the observation of spin-valve signals and Hanle signals in a nonlocal configuration. ${ }^{6}$ The observation of both signals provides direct evidence of spin injection and transport. Salis et al. investigated the temperature dependence of the spin signal in $\mathrm{Fe} / \mathrm{GaAs}$ and reported achieving spin injection at room temperature. ${ }^{7}$ We also achieved spin injection in $\mathrm{Co}_{50} \mathrm{Fe}_{50}$ $(\mathrm{CoFe}) / n$-GaAs Schottky tunnel junctions with an improved temperature dependence. ${ }^{12}$

Along with spin injection and detection, the understanding of spin interactions in semiconductors, such as spin-orbit, hyperfine, or spin-exchange coupling, is an important part of spintronics research. In particular, the interplay between electron spins and nuclear spins due to the hyperfine interaction has been studied extensively for application to quantum information devices based on nuclear spins as well as for understanding the transport properties of electron spins in semiconductors. ${ }^{13-17}$ The hyperfine interaction transfers the angular momentum from polarized electrons to nuclei, leading to an effective polarization of nuclear spins, referred to as dynamic nuclear polarization (DNP). In contrast, polarized nuclear spins affect electron spins as an effective magnetic field or Overhauser field. Thus, one can evaluate the degree of nuclear spin polarization through the strength of the Overhauser field. To detect the nuclear field, oblique Hanle effect measurements have been widely used, and both optical detection through a spin-LED with an Fe electrode ${ }^{18,19}$ and recently electrical detection through a lateral spin-transport device $^{20-22}$ with an $\mathrm{Fe}$ or $(\mathrm{Ga}, \mathrm{Mn}) \mathrm{As}$ electrode have been demonstrated.
Highly spin-polarized ferromagnetic material is indispensable for creating highly spin-polarized states of both electrons and nuclei. Co-based Heusler alloys are one candidate for a highly polarized spin source because of the half-metallic ferromagnetic nature theoretically predicted for many of these alloys $^{23,24}$ and because of their high Curie temperatures, which are well above room temperature. There have been several reports on spin injection through spin-LEDs with Co-based Heusler alloy electrodes. ${ }^{25-27}$ The reported spin-injection efficiency of these devices, however, has been less than that with an Fe electrode. Moreover, there has been no report on the electrical detection of spin injection using a lateral spin-transport device with a Heusler alloy electrode. Thus, the applicability of Co-based Heusler alloys to spin injection has not been fully clarified.

Recently, we found that fully epitaxial $\mathrm{Co}_{2} \mathrm{MnSi} /$ $\mathrm{MgO} / \mathrm{Co}_{2} \mathrm{MnSi}$ magnetic tunnel junctions (MTJs) with Mnrich $\mathrm{Co}_{2} \mathrm{MnSi}$ electrodes exhibit high tunneling magnetoresistance (TMR) ratios of up to $1995 \%$ at $4.2 \mathrm{~K}$ and up to $354 \%$ at $290 \mathrm{~K}^{28,29}$ The observed TMR ratios for MTJs with Mn-rich $\mathrm{Co}_{2} \mathrm{MnSi}$ electrodes is attributed to suppressed $\mathrm{Co}_{\mathrm{Mn}}$ antisites, which cause a reduced density of minority-spin in-gap states around $E_{F} \cdot{ }^{28,29}$ Furthermore, we reported high TMR ratios of $1135 \%$ at $4.2 \mathrm{~K}$ and $443 \%$ at $290 \mathrm{~K}$ in an MTJ having a $\mathrm{Co}_{2} \mathrm{MnSi}$ upper electrode via an ultrathin $\mathrm{CoFe}$ insertion layer on a $\mathrm{MgO}$ tunnel barrier in combination with a $\mathrm{CoFe}$ lower electrode-i.e., a $\mathrm{Co}_{2} \mathrm{MnSi}$ upper electrode/CoFe insertion layer/MgO barrier/CoFe lower electrode MTJ (Ref. 30) indicating a large spin polarization for $\mathrm{Co}_{2} \mathrm{MnSi}$. Thus, $\mathrm{Co}_{2} \mathrm{MnSi} / \mathrm{CoFe}$ bilayers are promising for effectively creating and detecting polarized spin states and the Overhauser field in semiconductors.

The purpose of this study is twofold. One purpose is to demonstrate spin injection from the Heusler alloy $\mathrm{Co}_{2} \mathrm{MnSi}$ into $\mathrm{GaAs}$ in a four-terminal nonlocal device with $\mathrm{Co}_{2} \mathrm{MnSi} / \mathrm{CoFe} / n$-GaAs Schottky tunnel junctions, in which $\mathrm{Co}_{2} \mathrm{MnSi}$ works as a spin source. The second purpose is to understand the transient behavior of the nuclear spins in GaAs channels. For the second purpose, we have investigated the Overhauser field produced by the DNP through the observation of transient oblique Hanle signals in $\mathrm{Co}_{2} \mathrm{MnSi} / \mathrm{CoFe} / n-\mathrm{GaAs}$ 
Schottky tunnel junctions with a four-terminal nonlocal configuration. In this study, we will show that samples with $\mathrm{Co}_{2} \mathrm{MnSi}$ electrodes exhibit a larger spin signal and a larger Overhauser field than those of reference samples with $\mathrm{CoFe}$ electrodes.

This paper is organized as follows. Section II describes our experimental methods. In Sec. III A, we demonstrate all electrical injection and detection of spin-polarized electrons through the observation of spin-valve signals and Hanle signals in a four-terminal nonlocal geometry. In Sec. III B, we compare the bias voltage-dependent spin polarization between $\mathrm{Co}_{2} \mathrm{MnSi} / \mathrm{CoFe} / n$-GaAs and $\mathrm{CoFe} / n$-GaAs samples and discuss the spin-injection efficiency of the $\mathrm{Co}_{2} \mathrm{MnSi}$ electrode. In Sec. III C, we show the transient oblique Hanle signals and discuss the model for the Overhauser field in a transient state. While the electrical detection of oblique Hanle signals has been done only in a steady state, we observe oblique Hanle signals in a transient state, in which the magnetic field is swept faster than the relaxation time of the nuclear spins. This gives us an important insight towards an understanding of nuclear spin dynamics, especially, the transient response of nuclear spins to a change in the magnetic field, and the characteristic time needed for the nuclear spins to reach a steady state through the DNP.

\section{EXPERIMENTAL METHODS}

Layer structures consisting of (from the substrate side) a 250-nm-thick undoped GaAs buffer layer, a 2.5- $\mu \mathrm{m}$-thick $n^{-}$GaAs channel layer, a 15 -nm-thick $n^{-} \rightarrow n^{+}$-GaAs transition layer, and a 15 -nm-thick $n^{+}$-GaAs layer were grown by molecular beam epitaxy at $590^{\circ} \mathrm{C}$ on semi-insulating $\mathrm{GaAs}(001)$ substrates. The doping concentration of the $n^{-}$-GaAs channel was chosen to be $3 \times 10^{16} \mathrm{~cm}^{-3}$, and the doping concentration of the $n^{+}$-GaAs was $5 \times 10^{18} \mathrm{~cm}^{-3}$ to form a narrow Schottky barrier. The samples were then capped with an arsenic protective layer and transported in air to an ultrahigh vacuum chamber capable of magnetron sputtering. After the arsenic cap was removed by heating the samples to $300^{\circ} \mathrm{C}$, a $1.1-\mathrm{nm}$ thick ultrathin $\mathrm{CoFe}$ layer and a 5-nm-thick $\mathrm{Co}_{2} \mathrm{MnSi}$ layer were deposited by magnetron sputtering at room temperature and successively annealed in situ at $350^{\circ} \mathrm{C}$. The $\mathrm{Co}_{2} \mathrm{MnSi}$ layer was deposited by cosputtering from a $\mathrm{Co}_{2} \mathrm{MnSi}$ target and a $\mathrm{Mn}$ target. The film composition of the $\mathrm{Co}_{2} \mathrm{MnSi}$ film was chosen to be $\mathrm{Co}_{2} \mathrm{Mn}_{1.30} \mathrm{Si}_{0.88}$ to suppress harmful $\mathrm{Co}_{\mathrm{Mn}}$ antisites. The ultrathin $\mathrm{CoFe}$ layer was inserted to improve the structural quality of the $\mathrm{Co}_{2} \mathrm{MnSi}$ layer. Last, a 5-nm-thick $\mathrm{Ru}$ cap layer was deposited using magnetron sputtering at room temperature. Using electron beam lithography and Ar ionmilling techniques, lateral spin-transport devices, as shown in Fig. 1, were fabricated. The size of the injector contact (contact-2) and detector contact (contact-3) were $0.5 \times 10 \mu \mathrm{m}$ and $1.0 \times 10 \mu \mathrm{m}$, respectively, and the spacing $(d)$ between them was 0.5 or $4.0 \mu \mathrm{m}$. We defined the longer direction of the junction as the $y$ axis direction. The sample was evaluated in a four-terminal nonlocal geometry in which the nonlocal voltage between contact -3 and contact- 4 was measured under a constant current $(I)$ supplied between contact- 2 and contact-1 at $4.2 \mathrm{~K}$ as functions of the in-plane magnetic field for spin-valve effect measurements, the out-of-plane magnetic field for Hanle effect measurements, and the oblique magnetic

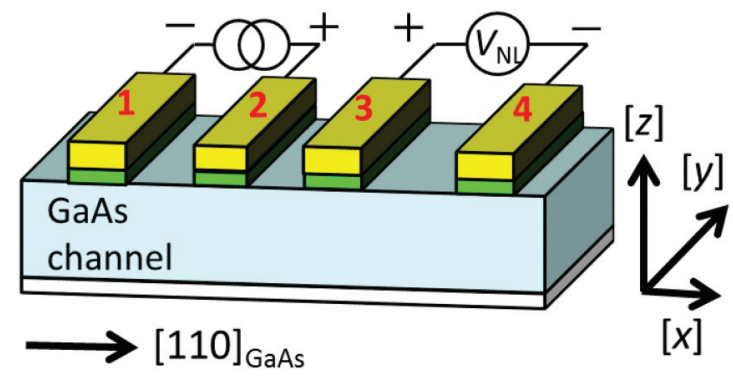

FIG. 1. (Color online) Schematic device structure of a fourterminal lateral spin-transport device and circuit configuration for nonlocal measurements.

field for oblique Hanle effect measurements. The bias voltage was defined with respect to the $n$-GaAs. As a reference, samples with a 5-nm-thick CoFe single layer were identically fabricated with the same conditions.

\section{RESULTS AND DISCUSSION}

\section{A. Spin-valve effect and Hanle effect}

From the measurements of the tunneling anisotropic magnetoresistance effect ${ }^{31-34}$ for both injector and detector contacts (not shown), we found that the easy axis of the magnetization of the $\mathrm{Co}_{2} \mathrm{MnSi} / \mathrm{CoFe}$ bilayer was along the shorter direction of the junction (the $x$ axis direction or $\left.[110]_{\mathrm{GaAs}}\right)$. Thus, the strong uniaxial-type magnetocrystalline anisotropy along the $x$ axis direction and relatively weak shape anisotropy along the $y$ axis direction were induced in the $\mathrm{Co}_{2} \mathrm{MnSi} / \mathrm{CoFe}$ bilayer. There have been several reports that ferromagnetic thin films, including Co-based Heusler alloys, epitaxially grown on $\mathrm{GaAs}(001)$ substrates have an uniaxial-type anisotropy with an easy axis of either the $[110]_{\mathrm{GaAs}}$ or $[1-10]_{\mathrm{GaAs}}$ direction ${ }^{35-38}$ and whose strength and direction depend on the surface reconstruction superstructures of GaAs and/or ferromagnet materials. In this study, the fabricated $\mathrm{Co}_{2} \mathrm{MnSi} / \mathrm{CoFe} / n$-GaAs junction had an easy axis of the $[110]_{\mathrm{GaAs}}$ direction, which corresponded to the $x$ axis direction. On the other hand, the $\mathrm{CoFe} / n$-GaAs junction of the reference sample had an easy axis of the $[1-10]_{\mathrm{GaAs}}$ direction, which corresponded to the $y$ axis direction.

Figure 2 shows a plot of nonlocal voltage as a function of the in-plane magnetic field $\left(B_{x}\right)$ for a lateral spin-transport device with $\mathrm{Co}_{2} \mathrm{MnSi} / \mathrm{CoFe} / n$-GaAs Schottky tunnel junctions. The magnetic field was applied along the $x$ axis direction, which corresponded to the easy axis for the magnetization of the $\mathrm{Co}_{2} \mathrm{MnSi} / \mathrm{CoFe}$ bilayer. A bias current $(I)$ of $-40 \mu \mathrm{A}$ was supplied, where electron spins were injected from the $\mathrm{Co}_{2} \mathrm{MnSi}$ to $n$-GaAs. We subtracted a background signal consisting of a constant term and linear and quadratic terms of $B_{x}$ so that the nonlocal voltage at $B_{x}=0$ was set to $0 \mathrm{~V}$ because electron spins are completely depolarized at $B_{x}=0$ due to precession by the Overhauser field, as will be explained in Sec. III C. Although a similar background signal was observed in identical experiments, ${ }^{6,8}$ its origin is still not well understood. A clear spin-valve-like change of the nonlocal voltage was observed at $B_{x} \cong+25$ and $-30 \mathrm{mT}$ due to switching between the parallel (P) and antiparallel (AP) states 


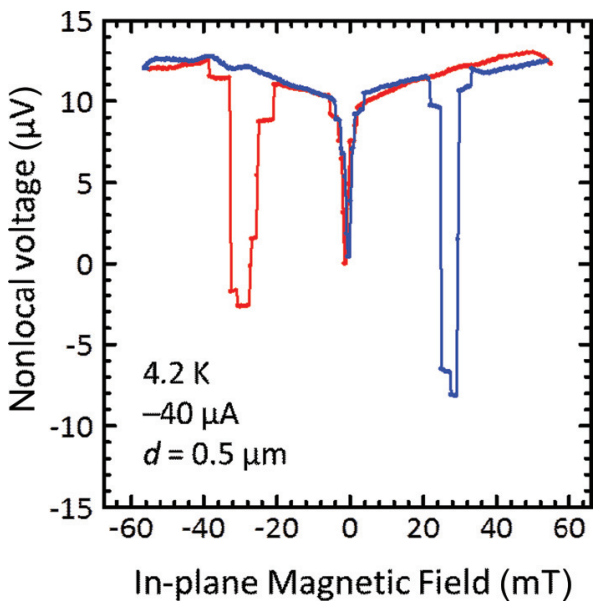

FIG. 2. (Color online) Plot of nonlocal voltage as a function of in-plane magnetic field $\left(B_{x}\right)$ for a lateral spin-transport device with $\mathrm{Co}_{2} \mathrm{MnSi} / \mathrm{CoFe} / n$-GaAs junctions. A background signal consisting of a constant term and linear and quadratic terms of $B_{x}$ has been subtracted.

for the magnetization configuration between the injector and detector contacts. However, a number of small steps were seen in the observed spin-valve signal. Furthermore, the magnetic field range for the AP state was relatively narrow, and $\left|V_{\mathrm{AP}}\right|$ was smaller than $\left|V_{\mathrm{P}}\right|$, suggesting an imperfect formation of the AP state. Here, $V_{\mathrm{P}}$ and $V_{\mathrm{AP}}$ are the nonlocal voltages for the $\mathrm{P}$ and AP states, respectively, and should have the same magnitude with an opposite sign. ${ }^{39,40}$ A possible origin of the small steps in the spin-valve signal and the imperfect formation of the AP state is a complex magnetization reversal arising from a conflict in the magnetic anisotropy between the shape anisotropy having the easy axis along the $y$ axis direction and the magnetocrystalline anisotropy having the easy axis along the $x$ axis direction.

Figure 3 shows a plot of nonlocal voltage as a function of out-of-plane magnetic field $\left(B_{z}\right)$ for both the $\mathrm{P}$ and AP configurations. The nonlocal voltage for the $\mathrm{P}(\mathrm{AP})$ configuration

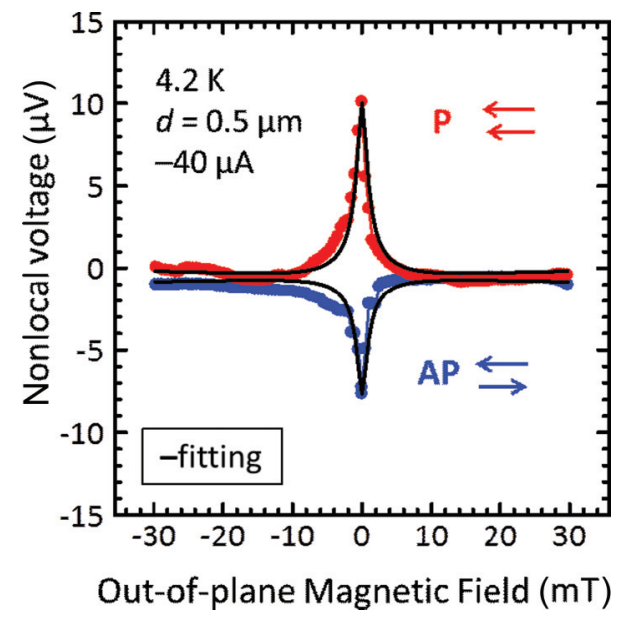

FIG. 3. (Color online) Plot of nonlocal voltage as a function of out-of-plane magnetic field $\left(B_{\mathrm{z}}\right)$ for a $\mathrm{Co}_{2} \mathrm{MnSi} / \mathrm{CoFe} / n$-GaAs sample for both $\mathrm{P}$ (upper curve) and AP (lower curve) configurations. The solid lines are the result of the fitting using Eq. (1). gradually decreased (increased) as $\left|B_{z}\right|$ increased, and the two curves merged at a large $B_{z}$. These results clearly indicate the Hanle effect. In the Hanle effect, the nonlocal voltage as a function of $B_{z}$ can be expressed by ${ }^{39,40}$

$$
\begin{aligned}
V_{\mathrm{NL}}\left(B_{Z}\right)= & \pm P_{\mathrm{inj}} \cdot P_{\mathrm{det}}\left(\frac{\rho l_{\mathrm{SF}}}{S}\right)\left(\frac{l_{\mathrm{SF}}}{\tau_{\mathrm{SF}}}\right) I \int_{0}^{\infty} \frac{1}{\sqrt{4 \pi D t}} \\
& \times \exp \left(-\frac{d^{2}}{4 D t}\right) \cos \left(\omega_{L} t\right) \exp \left(-\frac{t}{\tau_{\mathrm{SF}}}\right) d t,
\end{aligned}
$$

where $P_{\text {inj(det) }}$ is the spin polarization of the injector (detector) contact, $\rho$ is the resistivity of the GaAs channel, $S$ is the area of the channel cross section, $l_{\mathrm{SF}}$ is the spin-diffusion length, $d$ is the distance between contact- 2 and contact- $3, \tau_{\mathrm{SF}}$ is the spin lifetime, $I$ is the injected current, $D=l^{2} \mathrm{SF} / \tau_{\mathrm{SF}}$ is the diffusion constant, $\omega_{L}=g \mu_{B} B / \hbar$ is the Larmor frequency, $g$ $(=-0.44)$ is an electron $g$ factor for GaAs, $\mu_{\mathrm{B}}$ is the Bohr magneton, and $\hbar$ is the reduced Planck's constant. The $+(-)$ sign on the right-hand side of Eq. (1) corresponds to the P (AP) configuration. The observed Hanle curves can be fitted well with Eq. (1). The estimated spin lifetime $\tau_{\mathrm{SF}}$ was $50 \mathrm{~ns}$. This value of the spin lifetime is comparable to those obtained for GaAs with a doping concentration of $10^{16} \mathrm{~cm}^{-3}$ (Ref. 41), suggesting that spins were injected into the GaAs channel. The estimated spin diffusion length $l_{\mathrm{SF}}$ was $5 \mu \mathrm{m}$. The Hanle signal for the $\mathrm{P}$ state was approximately $10 \mu \mathrm{V}$, and that for the AP state was approximately $8 \mu \mathrm{V}$, so the total of both signals was approximately $18 \mu \mathrm{V}$. This value agrees with the result of the spin-valve effect. The smaller amplitude of the Hanle curve for the AP state was due to the imperfect formation of the AP state, as previously described. The observation of the spin-valve signal and Hanle signal in the four-terminal nonlocal geometry provides direct evidence of the spin injection, detection, and transport in GaAs.

\section{B. Comparison of spin polarization between $\mathrm{Co}_{2} \mathrm{MnSi}$ and $\mathrm{CoFe}$}

In this section, we compare the magnitude of the spin signal $\left(\left|V_{\mathrm{P}}\right|\right)$ and spin polarization between the sample with a $\mathrm{Co}_{2} \mathrm{MnSi}$ electrode and that with a $\mathrm{CoFe}$ electrode. Figure 4 shows the bias current dependence of $\left|V_{\mathrm{P}}\right|$ for both a $\mathrm{Co}_{2} \mathrm{MnSi} / \mathrm{CoFe} / n$-GaAs sample and a $\mathrm{CoFe} / n$-GaAs sample. The negative (positive) bias region corresponds to the spin injection (extraction), where spin-polarized electrons tunnel from ferromagnet (semiconductor) to semiconductor (ferromagnet). Here, the spacing $(d)$ between the injector and detector contacts was $4.0 \mu \mathrm{m}$ for both samples. The bias current dependence of $\left|V_{\mathrm{P}}\right|$ clearly differed between the $\mathrm{Co}_{2} \mathrm{MnSi} / \mathrm{CoFe} / n$-GaAs sample and the $\mathrm{CoFe} / n$-GaAs sample. For the $\mathrm{Co}_{2} \mathrm{MnSi} / \mathrm{CoFe} / n$-GaAs sample, $\left|V_{\mathrm{P}}\right|$ increased monotonically as $|I|$ increased in the negative-bias region, while no clear spin signal was observed in the positive-bias region. On the other hand, for the $\mathrm{CoFe} / n$-GaAs sample, $\left|V_{\mathrm{P}}\right|$ increased with increasing $|I|$, reached a peak value at $I=-25 \mu \mathrm{A}$, and then decreased with increasing $|I|$ in the negative-bias region, while it increased monotonically with increasing $|I|$ in the positive-bias region. By integrating Eq. (1) under $B_{\mathrm{Z}}=0,\left|V_{\mathrm{P}}\right|$ is given by

$$
V_{\mathrm{P}}=\frac{1}{2} P_{\mathrm{inj}} \cdot P_{\mathrm{det}}\left(\frac{\rho l_{\mathrm{SF}}}{S}\right) \exp \left(-\frac{d}{l_{\mathrm{SF}}}\right) I .
$$




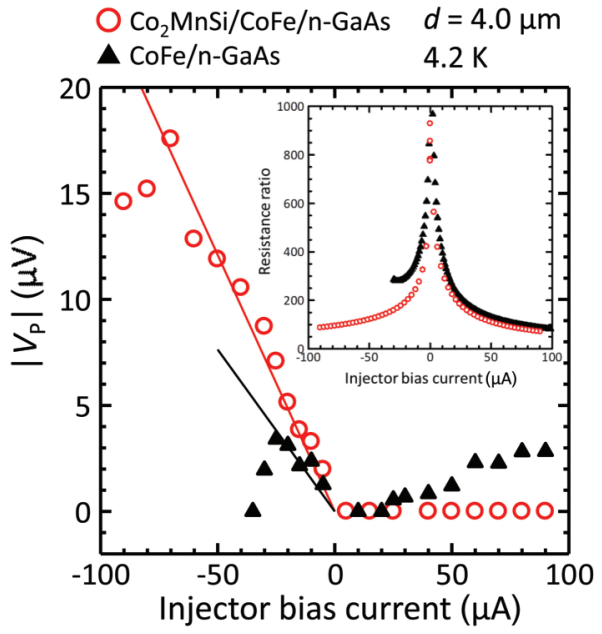

FIG. 4. (Color online) Bias current dependence of $V_{\mathrm{P}}$ measured for a $\mathrm{Co}_{2} \mathrm{MnSi} / \mathrm{CoFe} / n$-GaAs sample and a $\mathrm{CoFe} / n$-GaAs reference sample. The solid lines indicate linear least-squares fitting in the low negative-bias region. Inset shows the bias current dependence of the injector junction resistance for both $\mathrm{Co}_{2} \mathrm{MnSi} / \mathrm{CoFe} / n$-GaAs and $\mathrm{CoFe} / n$-GaAs. The junction resistance was normalized by the spin resistance of the GaAs channel.

The above equation is valid when the junction resistance is much larger than the spin resistance of the channel, which condition is satisfied in our devices, as shown later. Thus, $\left|V_{\mathrm{P}}\right|$ depends on $I, P_{\mathrm{inj}(\mathrm{det})}, \rho, S, l_{\mathrm{SF}}$, and $d$. Since the channel structure of GaAs is the same for both samples, the values of $\rho$, $S, l_{\mathrm{SF}}$, and $d$ are the same. Therefore, the difference in the $\left|V_{\mathrm{P}}\right|$ vs $I$ characteristics between the two samples comes from the difference in the $P_{\text {inj }} \cdot P_{\text {det }}$ products. This result indicates that the spin polarization at the $1.1-\mathrm{nm}$-thick $\mathrm{CoFe} / \mathrm{GaAs}$ interface of $\mathrm{Co}_{2} \mathrm{MnSi} / \mathrm{CoFe}(1.1 \mathrm{~nm}) / \mathrm{GaAs}$ heterojunction differs from that at the 5-nm-thick $\mathrm{CoFe} / \mathrm{GaAs}$ interface, which means that $\mathrm{Co}_{2} \mathrm{MnSi}$ works as a spin source in the $\mathrm{Co}_{2} \mathrm{MnSi} / \mathrm{CoFe} / n$ GaAs sample even though 1.1-nm-thick CoFe was inserted between the $\mathrm{Co}_{2} \mathrm{MnSi}$ and GaAs. This is reasonable because the thickness of the inserted $\mathrm{CoFe}(1.1-\mathrm{nm})$ layer was less than the spin diffusion length of $\mathrm{CoFe}(\sim 10 \mathrm{~nm}){ }^{42}$

The nonlinear relations between $\left|V_{\mathrm{P}}\right|$ and $I$ shown in Fig. 4 indicate that the value of $\left|P_{\text {inj }} \cdot P_{\text {det }}\right|^{1 / 2}$ is not constant against the bias voltage. There have been several experimental ${ }^{6,7,12}$ and theoretical ${ }^{43-45}$ investigations into whether the magnitude and sign of the spin polarization for a ferromagnet/GaAs heterointerface are affected by the bias condition. Although the junction resistance also affects the spin-injection efficiency, ${ }^{46}$ the effect was negligible in our devices. The inset in Fig. 4 shows the junction resistance of the injector contacts of both $\mathrm{CoFe}$ and $\mathrm{Co}_{2} \mathrm{MnSi} / \mathrm{CoFe}$ as a function of the bias current. The junction resistance was normalized by the spin resistance of the GaAs channel, $\rho l_{\mathrm{SF}} / S$. As shown in the inset, the junction resistance is at least two orders of magnitude larger than the spin resistance for almost all bias regions investigated in this study. Thus, $V_{\mathrm{P}}$ does not depend on the junction resistance, as indicated by Eq. (2), and the nonlinear relations between $\left|V_{\mathrm{P}}\right|$ and $I$ result from the change in the $P_{\text {inj }} \cdot P_{\text {det }}$ value by the bias voltage rather than from the change in the junction resistance. Table I summarizes the values of $\left|P_{\text {inj }} \cdot P_{\text {det }}\right|^{1 / 2}$ in a low negative-
TABLE I. Summary of $\left|P_{\text {inj }} \cdot P_{\text {det }}\right|^{1 / 2}$ in the low negative-bias region for a $\mathrm{Co}_{2} \mathrm{MnSi} / \mathrm{CoFe} / n$-GaAs sample and $\mathrm{CoFe} / n-\mathrm{GaAs}$ reference samples with and without annealing.

\begin{tabular}{lcc}
\hline \hline Sample structure & Annealing temperature $\left({ }^{\circ} \mathrm{C}\right)$ & $\left|P_{\text {inj }} \cdot P_{\text {det }}\right|^{1 / 2}(\%)$ \\
\hline $\mathrm{Co}_{2} \mathrm{MnSi} / \mathrm{CoFe} / n-$ & 350 & 4.4 \\
$\mathrm{GaAs}$ & & \\
$\mathrm{CoFe} / n-\mathrm{GaAs}$ & 350 & 3.5 \\
$\mathrm{CoFe} / n-\mathrm{GaAs}^{12}$ & - & 3.0 \\
\hline \hline
\end{tabular}

bias current region, in which the values of $\left|P_{\text {inj }} \cdot P_{\text {det }}\right|^{1 / 2}$ are almost constant. The value for a sample without postdeposition annealing $^{12}$ is also shown for comparison. Importantly, the value of $\left|P_{\text {inj }} \cdot P_{\text {det }}\right|^{1 / 2}$ for the $\mathrm{Co}_{2} \mathrm{MnSi} / \mathrm{CoFe} / n$-GaAs sample is larger than those for the $\mathrm{CoFe} / n$-GaAs samples. The larger value of $\left|P_{\text {inj }} \cdot P_{\text {det }}\right|^{1 / 2}$ obtained for the $\mathrm{Co}_{2} \mathrm{MnSi} / \mathrm{CoFe} / n$ GaAs sample cannot be explained by the difference of the junction resistance, because the junction resistances of both the samples were much larger than the spin resistance, resulting in the value of $\left|P_{\text {inj }} \cdot P_{\text {det }}\right|^{1 / 2}$ being independent of the junction resistance. These results indicate that $\mathrm{Co}_{2} \mathrm{MnSi}$ has higher spin polarization and that a Heusler alloy is a promising spin source for efficient spin injection into a semiconductor. Considering that the TMR ratios in MTJs with $\mathrm{Co}_{2} \mathrm{MnSi}$ electrodes increase with increasing annealing temperature around $550{ }^{\circ} \mathrm{C},{ }^{47}$ there is much room to improve the spin-injection efficiency with our sample. It should be noted, however, that high-temperature annealing may lead to interdiffusion of atomic species at a ferromagnet/GaAs interface, resulting in the degradation of spin-injection properties. Thus, the annealing temperature of the $\mathrm{Co}_{2} \mathrm{MnSi}$ layer must be optimized to simultaneously obtain high spin polarization and prevent interdiffusion.

\section{Oblique Hanle effect}

In this section, we describe the electrical detection of the Overhauser field through the observation of oblique Hanle signals. The steady-state Overhauser field $\left(\mathbf{B}_{\mathbf{n}}\right)$ induced by the DNP can be expressed by ${ }^{48}$

$$
\mathbf{B}_{\mathbf{n}}=f b_{n} \frac{\mathbf{B}_{\mathbf{o b}} \cdot \mathbf{S}}{\mathbf{B}_{\mathbf{o b}}^{2}+\xi B_{l}^{2}} \mathbf{B}_{\mathbf{o b}},
$$

where $f(\leqslant 1)$ is the leakage factor and $b_{n}$ is the effective field due to the polarization of nuclear spins, which takes the negative value of $-17 \mathrm{~T}$ in GaAs for the theoretical ideal case. $^{49} \mathbf{S}$ is the average electron spin $(|\mathbf{S}|=1 / 2$ corresponds to $\left.P_{\mathrm{GaAs}}=100 \%\right), \mathbf{B}_{\mathbf{o b}}$ is the external magnetic field, $B_{l}$ is the local dipolar field experienced by the nuclei, and $\xi$ is a numerical coefficient on the order of unity, which depends on the nature of the spin-spin interactions. ${ }^{48,49}$ In the oblique Hanle effect measurement, $\mathbf{B}_{\mathbf{o b}}$ is applied obliquely with respect to $\mathbf{S}$ so that electron spins make precession with $\mathbf{B}_{\mathbf{n}}$ and $\mathbf{B}_{\mathbf{o b}}$. Note that in the conventional Hanle effect measurement, in which $\mathbf{S}$ and $\mathbf{B}_{\mathbf{o b}}$ are orthogonal, no nuclear field is generated because $\mathbf{B}_{\mathbf{o b}} \cdot \mathbf{S}=0$. Figure 5(a) shows a simulated nonlocal voltage using Eqs. (1) and (3) as a function of $B_{\text {ob. Here, we }}$ set $\mathbf{S}=S \mathbf{x}$ and $\mathbf{B}_{\mathbf{o b}}=B_{\mathrm{ob}}(\mathbf{x} \sin \theta+\mathbf{z} \cos \theta)$, where $\mathbf{x}$ and $\mathbf{z}$ are unit vectors along the $x$ axis and $z$ axis directions, respectively. Considering that electron spins experience the total magnetic 

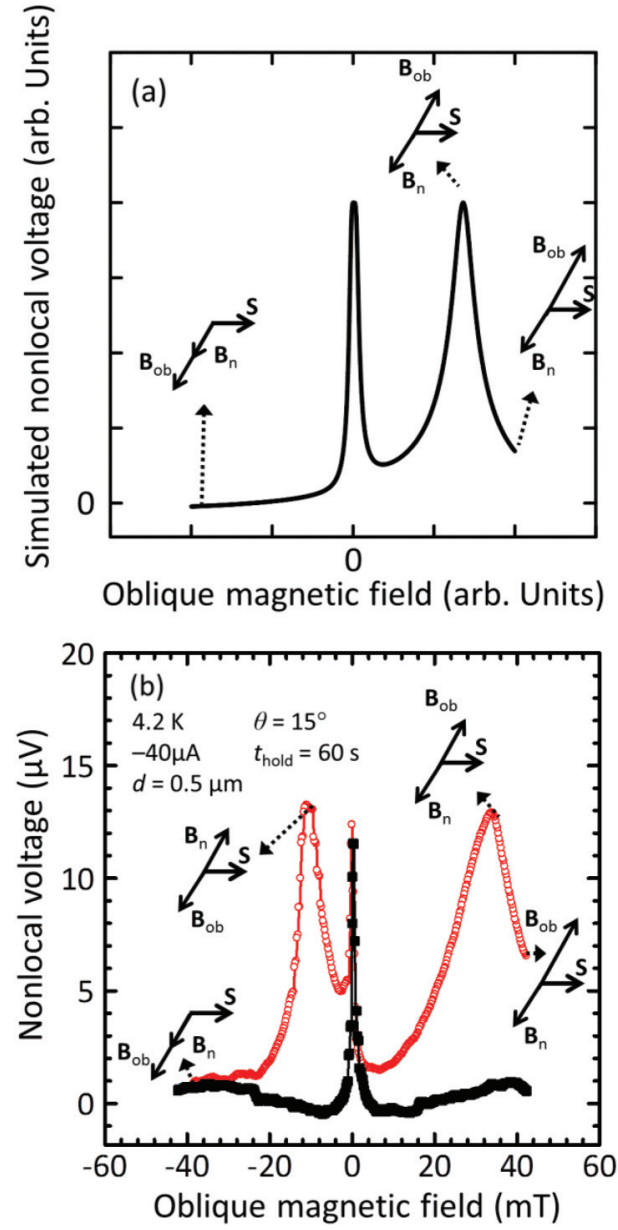

FIG. 5. (Color online) (a) Simulated nonlocal voltage in the oblique Hanle effect using Eqs. (1) and (3) as a function of oblique magnetic field $\left(B_{\mathrm{ob}}\right)$. The vector diagrams show the relative orientation of the average electron spin $\mathbf{S}$, the oblique magnetic field $\mathbf{B}_{\mathbf{o b}}$, and the nuclear field $\mathbf{B}_{\mathbf{n}}$. (b) $B_{\mathrm{ob}}$ dependence of the nonlocal voltage for the $\mathrm{Co}_{2} \mathrm{MnSi} / \mathrm{CoFe} / n$-GaAs sample in the $\mathrm{P}$ magnetization configuration. The magnetic field was swept from +42 to $-42 \mathrm{mT}$ (open circle) and was swept back from -42 to +42 $\mathrm{mT}$ (black square) with a sweep rate of $0.18 \mathrm{mT} / \mathrm{s}$.

field of $\mathbf{B}_{\mathbf{o b}}+\mathbf{B}_{\mathbf{n}}$, and $b_{n}$ is negative, the behavior of the nonlocal voltage shown in Fig. 5(a) can be explained as follows. At $B_{\mathrm{ob}}>0, \mathbf{B}_{\mathbf{n}}$ and $\mathbf{B}_{\mathbf{o b}}$ are antiparallel, and electron spins experience a smaller effective magnetic field than $\left|\mathbf{B}_{\mathbf{o b}}\right|$. When $\mathbf{B}_{\mathbf{n}}$ and $\mathbf{B}_{\mathbf{o b}}$ cancel each other, electron spins get polarized and the nonlocal voltage shows a satellite peak. At $B_{\text {ob }}<0$, on the other hand, no satellite peak appears, because $\mathbf{B}_{\mathbf{n}}$ and $\mathbf{B}_{\mathbf{o b}}$ are parallel, and no cancelation occurs. Since it takes several seconds or more for the nuclear field to reach the steady state, ${ }^{18-20}$ very slow sweeping of the external magnetic field is necessary for the steady-state measurement.

Figure 5(b) shows the $B_{\mathrm{ob}}$ dependence of the nonlocal voltage for a $\mathrm{Co}_{2} \mathrm{MnSi} / \mathrm{CoFe} / n$-GaAs sample in the parallel magnetization configuration. Since $\mathbf{S}$ is parallel to the $x$ axis direction in the $\mathrm{Co}_{2} \mathrm{MnSi} / \mathrm{CoFe} / n$-GaAs sample, we applied $\mathbf{B}_{\mathbf{o b}}$ along the direction oblique by $15^{\circ}$ from the $z$ axis in the $x-z$ plane so that electron spins were affected by $\mathbf{B}_{\mathbf{o b}}$ and $\mathbf{B}_{\mathbf{n}}$. The device was first initialized at $B_{\mathrm{ob}}=+42 \mathrm{mT}$ for a hold time
( $t_{\text {hold }}$ ) of $60 \mathrm{~s}$ at an injection current of $-40 \mu \mathrm{A}$ so that nuclear spins became dynamically polarized. The magnetic field was then swept from +42 to $-42 \mathrm{mT}$ (negative sweep direction) and was swept back from -42 to $+42 \mathrm{mT}$ (positive sweep direction), with a sweep rate of $0.18 \mathrm{mT} / \mathrm{s}$. This sweep rate was too fast for the nuclear field to reach the steady state. Compared to the steady-state signal, the observed transient oblique Hanle signal has two features: (1) an additional satellite peak is observed at $-10 \mathrm{mT}$ in the negative sweep direction, and (2) no satellite peak is observed in the positive sweep direction, showing a clear hysteretic nature depending on the sweep direction.

To explain the observed oblique Hanle signal, we discuss the behavior of the nuclear spin in the transient state. In the negative sweep direction, the behavior of the nuclear field for $B_{\mathrm{ob}}>0$ is qualitatively similar to that for the steadystate nuclear field; i.e., the nuclear field is generated along the AP direction to $\mathbf{B}_{\mathbf{o b}}$ during an initial holding time at $B_{\mathrm{ob}}=+42 \mathrm{mT}$, and then $\mathbf{B}_{\mathbf{o b}}$ and $\mathbf{B}_{\mathbf{n}}$ cancel each other at $B_{\mathrm{ob}}=+33 \mathrm{mT}$. In a similar way, the observation of the satellite peak at $B_{\mathrm{ob}}=-10 \mathrm{mT}$ indicates that electron spins are repolarized due to the cancellation of $\mathbf{B}_{\mathbf{o b}}$ and $\mathbf{B}_{\mathbf{n}}$. However, this AP state for $\mathbf{B}_{\mathbf{n}}$ with respect to $\mathbf{B}_{\mathbf{o b}}$ is the transient state because $\mathbf{B}_{\mathbf{n}}$ and $\mathbf{B}_{\mathbf{o b}}$ are in a $\mathrm{P}$ configuration at $B_{\mathrm{ob}}<0$ in the steady state, as indicated by Eq. (3). Then, $\mathbf{B}_{\mathbf{n}}$ gradually goes to the steady state, and it reaches almost the steady state at $B_{\mathrm{ob}}=-42 \mathrm{mT}$, resulting in being parallel to $\mathbf{B}_{\mathbf{o b}}$. In the positive sweep direction, $\mathbf{B}_{\mathbf{o b}}$ and $\mathbf{B}_{\mathbf{n}}$ are parallel at $B_{\mathrm{ob}}<0$, and this $\mathrm{P}$ configuration is transiently kept just after $\mathbf{B}_{\mathbf{o b}}$ is reversed from the negative direction to the positive one. Thus, no cancellation occurs between $\mathbf{B}_{\mathbf{o b}}$ and $\mathbf{B}_{\mathbf{n}}$, resulting in the disappearance of the satellite peak at $B_{\mathrm{ob}}>0$ for the positive sweep direction.

As discussed above, the transient oblique Hanle signals observed in the $\mathrm{Co}_{2} \mathrm{MnSi} / \mathrm{CoFe} / n$-GaAs sample can be qualitatively explained by adiabatic nuclear spin reversal; that is, the nuclear spins adiabatically rotate by $180^{\circ}$ when the magnetic field crosses zero. ${ }^{19,21}$ A similar adiabatic nuclear spin reversal has been reported in the electrical detection of spin-valve signals for lateral spin-transport devices with $\mathrm{Fe} / \mathrm{GaAs}$ Schottky tunnel junctions ${ }^{21}$ and in the optical detection of oblique Hanle signals for a spin-LED with a MnSb ferromagnet electrode. ${ }^{19}$ However, there has been no report on the transient behavior of the nuclear spins through the electrical detection of oblique Hanle signals. In this study, we have shown that adiabatic nuclear spin reversal with the magnetic field is an important factor in understanding transient oblique Hanle signals.

In the spin-valve signal measurement for the $\mathrm{Co}_{2} \mathrm{MnSi} / \mathrm{CoFe} / n$-GaAs sample, the dip structure was observed at $B_{x} \cong 0$, as shown in Fig. 2, indicating that electron spins were depolarized. This contrasted with the result for $\mathrm{CoFe} / n$-GaAs samples, in which the dip structure was not clearly observed and the spin-valve signal was almost unchanged from $V_{\mathrm{P}}$ at a zero in-plane magnetic field. ${ }^{12}$ For the $\mathrm{Co}_{2} \mathrm{MnSi} / \mathrm{CoFe} / n$-GaAs sample, the Overhauser field with its direction parallel or antiparallel to $\mathbf{B}_{\mathbf{x}}$ was generated at a sufficiently large $\left|\mathbf{B}_{\mathbf{x}}\right|$. Since the magnetization of the $\mathrm{Co}_{2} \mathrm{MnSi} / \mathrm{CoFe}$ electrode oriented to the $x$ axis direction, the stray field from the $\mathrm{Co}_{2} \mathrm{MnSi} / \mathrm{CoFe}$ electrode penetrated almost vertically into the GaAs channel. Thus, the Overhauser 


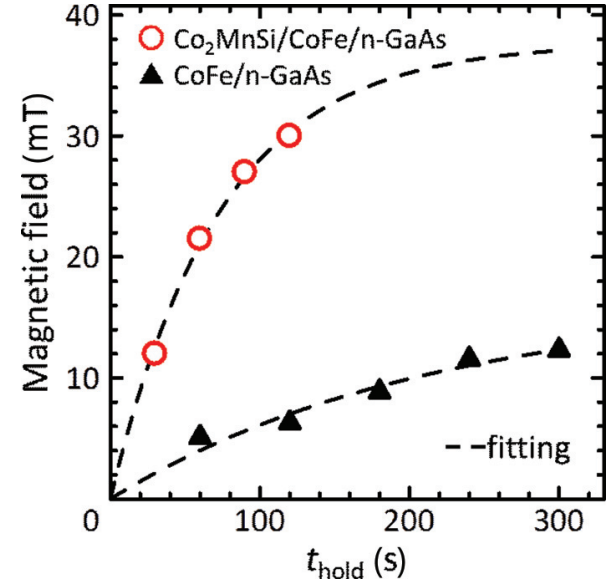

FIG. 6. (Color online) The $t_{\text {hold }}$ dependence of the observed satellite peak position at $B_{\mathrm{ob}}>0$ for a $\mathrm{Co}_{2} \mathrm{MnSi} / \mathrm{CoFe} / n-\mathrm{GaAs}$ sample and a $\mathrm{CoFe} / n$-GaAs sample. The satellite peak position shows almost exponential dependence (dash line).

field dynamically rotated and oriented to the direction of the stray field at $B_{x}=0$, causing electron spins to be completely depolarized. Note that since the stray field is comparable to or smaller than $B_{l}$, the strength of the steady-state Overhauser field generated by the stray field as well as that of the stray field itself is too small for electron spins to be completely depolarized. On the other hand, for the $\mathrm{CoFe} / n$-GaAs sample, the stray field penetrated along the $y$ axis direction, since the magnetization of the $\mathrm{CoFe} / n-\mathrm{GaAs}$ electrode oriented to the $y$ axis direction. Thus, the direction of the Overhauser field oriented parallel or antiparallel to the $y$ axis direction, resulting in no electron spin precession occurring.

One can estimate the strength of the nuclear field from the oblique Hanle signal because $\mathbf{B}_{\mathbf{o b}}+\mathbf{B}_{\mathbf{n}}=0$ is satisfied at the satellite peak position. Furthermore, one can estimate through transient analysis the time scale needed for the nuclear spins to reach a steady state. Figure 6 shows the $t_{\text {hold }}$ dependence of the observed satellite peak position for $B_{\mathrm{ob}}>0$. The circles and triangles indicate the data for the $\mathrm{Co}_{2} \mathrm{MnSi} / \mathrm{CoFe} / n-\mathrm{GaAs}$ sample and those for the nonannealed $\mathrm{CoFe} / n$-GaAs sample, respectively. For both samples, the satellite peak position shows almost exponential dependence. From the rate equation for the DNP, the time evolution of the nuclear field is given by ${ }^{18,19}$

$$
B_{N}(t)=B_{N}(\infty)\left\{1-\exp \left[-t\left(\frac{1}{T_{P}}+\frac{1}{T_{1}}\right)\right]\right\},
$$

where $B_{N}(\infty)$ is the steady-state Overhauser field, which is given by Eq. (3) and $T_{P}^{-1}$ and $T_{1}^{-1}$ are the rates for nuclear polarization through DNP and that for nuclear spin relaxation through nuclear-lattice interaction, respectively. Importantly, the saturation value of the satellite peak position of $37 \mathrm{mT}$ for the $\mathrm{Co}_{2} \mathrm{MnSi} / \mathrm{CoFe} / n$-GaAs sample is larger than that of $16 \mathrm{mT}$ for the $\mathrm{CoFe} / n-\mathrm{GaAs}$ sample, suggesting a greater Overhauser magnetic field due to the higher spin polarization of $\mathrm{Co}_{2} \mathrm{MnSi}$. The characteristic time, $\left(1 / T_{P}+\right.$ $\left.1 / T_{1}\right)^{-1}$, estimated from the exponential dependence of the satellite peak positions on $t_{\text {hold }}$ is approximately $77 \mathrm{~s}$ for the $\mathrm{Co}_{2} \mathrm{MnSi} / \mathrm{CoFe} / n$-GaAs sample and $213 \mathrm{~s}$ for the $\mathrm{CoFe} / n$ GaAs sample, respectively. These values are reasonable for the time scale needed for the nuclear spins to reach the steady state through the DNP. ${ }^{18,19,21}$ The value of $|2 \mathbf{S}|$, which corresponds to the spin polarization in the channel, estimated from the saturation value of the Overhauser field, was $5.4 \%$ for the $\mathrm{Co}_{2} \mathrm{MnSi} / \mathrm{CoFe} / n$-GaAs sample and $2.3 \%$ for the $\mathrm{CoFe} / n$ GaAs sample, respectively. These values are comparable to the effective spin polarizations of $\left|P_{\text {inj }} \cdot P_{\text {det }}\right|^{1 / 2}$, estimated from the $\left|V_{\mathrm{P}}\right|$ for both samples (see Table I).

\section{CONCLUSION}

We achieved efficient spin injection and a resultant efficient DNP by using a $\mathrm{Co}_{2} \mathrm{MnSi}$ spin source. The maximum spin polarization of both electron spins and nuclear spins in the GaAs channel observed in the sample with $\mathrm{Co}_{2} \mathrm{MnSi}$ electrodes was larger than that observed in the reference sample with $\mathrm{CoFe}$ electrodes; this was due to higher spin polarization of $\mathrm{Co}_{2} \mathrm{MnSi}$. These results indicate that the Heusler alloy is a promising spin source for spin injection into a semiconductor.

\section{ACKNOWLEDGMENTS}

We would like to thank S. Adachi and R. Kaji of Hokkaido University for their valuable discussion on the dynamics of nuclear spins. This work was partly supported by Grants-inAid for Scientific Research (Japan Society for the Promotion of Science (JSPS) KAKENHI Grants No. 22560001 and No. 48123246055) and by the Murata Science Foundation. T.A. was also supported by a Research Fellowship for Young Scientists from the JSPS. *uemura@ist.hokudai.ac.jp

${ }^{1}$ H. J. Zhu, M. Ramsteiner, H. Kostial, M. Wassermeier, H.-P. Schönherr, and K. H. Ploog, Phys. Rev. Lett. 87, 016601 (2001).

${ }^{2}$ X. Jiang, R. Wang, R. M. Shelby, R. M. Macfarlane, S. R. Bank, J. S. Harris, and S. S. P. Parkin, Phys. Rev. Lett. 94, 056601 (2005). ${ }^{3}$ P. Renucci, V. G. Truong, H. Jaffrès, L. Lombez, P. H. Binh, T. Amand, J. M. George, and X. Marie, Phys. Rev. B 82, 195317 (2010)

${ }^{4}$ M. Yasar, R. Mallory, A. Petrou, A. T. Hanbicki, G. Kioseoglou, C. H. Li, O. M. J. van't Erve, and B. T. Jonker, Appl. Phys. Lett. 94, 032102 (2009).
${ }^{5}$ B. D. Schultz, N. Marom, D. Naveh, X. Lou, C. Adelmann, J. Strand, P. A. Crowell, L. Kronik, and C. J. Palmstrøm, Phys. Rev. B 80, 201309(R) (2009).

${ }^{6}$ X. Lou, C. Adelmann, S. A. Crooker, E. S. Garlid, J. Zhang, K. S. M. Reddy, S. D. Flexner, C. J. Palmstrøm, and P. A. Crowell, Nat. Phys. 3, 197 (2007).

${ }^{7}$ G. Salis, A. Fuhrer, R. R. Schlittler, L. Gross, and S. F. Alvarado, Phys. Rev. B 81, 205323 (2010).

${ }^{8}$ M. Ciorga, A. Einwanger, U. Wurstbauer, D. Schuh, W. Wegscheider, and D. Weiss, Phys. Rev. B 79, 165321 (2009). 
${ }^{9}$ T. Suzuki, T. Sasaki, T. Oikawa, M. Shiraishi, Y. Suzuki, and K. Noguchi, Appl. Phys. Express 4, 023003 (2011).

${ }^{10}$ Y. Zhou, W. Han, L.-T. Chang, F. Xiu, M. Wang, M. Oehme, I. A. Fischer, J. Schulze, R. K. Kawakami, and K. L. Wang, Phys. Rev. B 84, 125323 (2011).

${ }^{11}$ H. Kum, J. Heo, S. Jahangir, A. Banerjee, W. Guo, and P. Bhattacharya, Appl. Phys. Lett. 100, 182407 (2012).

${ }^{12}$ T. Uemura, T. Akiho, M. Harada, K-i. Matsuda, and M. Yamamoto, Appl. Phys. Lett. 99, 082108 (2011).

${ }^{13}$ H. Sanada, Y. Kondo, S. Matsuzaka, K. Morita, C. Y. Hu, Y. Ohno, and H. Ohno, Phys. Rev. Lett. 96, 067602 (2006).

${ }^{14}$ G. Yusa, K. Muraki, K. Takashina, K. Hashimoto, and Y. Hirayama, Nature 434, 1001 (2005).

${ }^{15}$ R. Kaji, S. Adachi, H. Sasakura, and S. Muto, Phys. Rev. B 77, 115345 (2008).

${ }^{16}$ P. Maletinsky, C. W. Lai, A. Badolato, and A. Imamoglu, Phys. Rev. B 75, 035409 (2007).

${ }^{17}$ D. Gammon, Al. L. Efros, T. A. Kennedy, M. Rosen, D. S. Katzer, D. Park, S. W. Brown, V. L. Korenev, and I. A. Merkulov, Phys. Rev. Lett. 86, 5176 (2001).

${ }^{18}$ J. Strand, X. Lou, C. Adelmann, B. D. Schultz, A. F. Isakovic, C. J. Palmstrøm, and P. A. Crowell, Phys. Rev. B 72, 155308 (2005).

${ }^{19}$ P. Van Dorpe, W. Van Roy, J. De Boeck, and G. Borghs, Phys. Rev. B 72, 035315 (2005).

${ }^{20}$ M. K. Chan, Q. O. Hu, J. Zhang, T. Kondo, C. J. Palmstrøm, and P. A. Crowell, Phys. Rev. B 80, 161206(R) (2009).

${ }^{21}$ G. Salis, A. Fuhrer, and S. F. Alvarado, Phys. Rev. B 80, 115332(R) (2009).

${ }^{22}$ J. Shiogai, M. Ciorga, M. Utz, D. Schuh, T. Arakawa, M. Kohda, K. Kobayashi, T. Ono, W. Wegscheider, D. Weiss, and J. Nitta, Appl. Phys. Lett. 101, 212402 (2012).

${ }^{23}$ S. Ishida, S. Fujii, S. Kashiwagi, and S. Asano, J. Phys. Soc. Jpn. 64, 2152 (1995)

${ }^{24}$ S. Picozzi, A. Continenza, and A. J. Freeman, Phys. Rev. B 66, 094421 (2002).

${ }^{25}$ X. Y. Dong, C. Adelmann, J. Q. Xie, C. J. Palmstrøma, X. Lou, J. Strand, P. A. Crowell, J.-P. Barnes, and A. K. Petford-Long, Appl. Phys. Lett. 86, 102107 (2005).

${ }^{26}$ M. C. Hickey, C. D. Damsgaard, I. Farrer, S. N. Holmes, A. Husmann, J. B. Hansen, C. S. Jacobsen, D. A. Ritchie, R. F. Lee, G. A. C. Jones, and M. Pepper, Appl. Phys. Lett. 86, 252106 (2005).

${ }^{27}$ R. Farshchi, P. Bruski, M. Ramsteiner, J. Herfort, O. Brandt, Y. Manzke, K.-J. Friedland, and H. T. Grahn, Solid State Commun. 151, 436 (2011).
${ }^{28}$ T. Ishikawa, H. Liu, T. Taira, K.-i. Matsuda, T. Uemura, and M. Yamamoto, Appl. Phys. Lett. 95, 232512 (2009).

${ }^{29}$ H.-x. Liu, Y. Honda, T. Taira, K.-i. Matsuda, M. Arita, T. Uemura, and M. Yamamoto, Appl. Phys. Lett. 101, 132418 (2012).

${ }^{30}$ H.-x. Liu, Y. Honda, T. Taira, T. Uemura, and M. Yamamoto (unpublished).

${ }^{31}$ C. Gould, C. Rüster, T. Jungwirth, E. Girgis, G. M. Schott, R. Giraud, K. Brunner, G. Schmidt, and L. W. Molenkamp, Phys. Rev. Lett. 93, 117203 (2004).

${ }^{32}$ A. Matos-Abiague and J. Fabian, Phys. Rev. B 79, 155303 (2009).

${ }^{33}$ T. Akiho, T. Uemura, M. Harada, K.-i. Matsuda, and M. Yamamoto, Appl. Phys. Lett. 98, 232109 (2011).

${ }^{34}$ T. Akiho, T. Uemura, M. Harada, K.-i. Matsuda, and M. Yamamoto, Jpn. J. Appl. Phys. 51, $02 B M 01$ (2012).

${ }^{35}$ S. Kawagishi, T. Uemura, Y. Imai, K.-i. Matsuda, and M. Yamamoto, J. Appl. Phys. 103, 07 A703 (2008).

${ }^{36}$ T. Yano, T. Uemura, K.-i. Matsuda, and M. Yamamoto, J. Appl. Phys. 101, 063904 (2007).

${ }^{37}$ T. Ambrose, J. J. Krebs, and G. A. Prinz, Appl. Phys. Lett. 76, 3280 (2000).

${ }^{38}$ W. H. Wang, X. B. Ren, G. H. Wu, M. Przybylski, J. Barthel, and J. Kirschner, IEEE Trans. Magn. 41, 2805 (2005).

${ }^{39}$ I. Zutic, J. Fabian, and S. D. Samra, Rev. Mod. Phys. 76, 323 (2004).

${ }^{40}$ J. Fabian, A. Matos-Abiaguea, C. Ertlera, P. Stano, and I. I. Zutic, Acta Phys. Slovaca 57, 565 (2007).

${ }^{41}$ R. I. Dzhioev, K. V. Kavokin, V. L. Korenev, M. V. Lazarev, B. Ya. Meltser, M. N. Stepanova, B. P. Zakharchenya, D. Gammon, and D. S. Katzer, Phys. Rev. B 66, 245204 (2002).

${ }^{42}$ C. Ahn, K.-H. Shin, R. Loloee, J. Bass, and W. P. Pratt, Jr., J. Appl. Phys. 108, 023908 (2010).

${ }^{43}$ H. Dery and L. J. Sham, Phys. Rev. Lett. 98, 046602 (2007).

${ }^{44}$ A. N. Chantis, K. D. Belashchenko, D. L. Smith, E. Y. Tsymbal, M. van Schilfgaarde, and R. C. Albers, Phys. Rev. Lett. 99, 196603 (2007).

${ }^{45}$ S. Honda, H. Itoh, and J. Inoue, J. Phys. D 43, 135002 (2010).

${ }^{46}$ A. Fert and H. Jaffrès, Phys. Rev. B 64, 184420 (2001).

${ }^{47}$ T. Ishikawa, S. Hakamata, T. Uemura, K.-i. Matsuda, and M. Yamamoto, J. Appl. Phys. 103, 07A919 (2008).

${ }^{48}$ Optical Orientation, edited by F. Meier and B. P. Zakharchenya (North-Holland, New York, 1984).

${ }^{49}$ D. Paget, G. Lampel, B. Sapoval, and V. I. Safarov, Phys. Rev. B 15, 5780 (1977). 